\title{
Study on the Hotel Customer Satisfaction Evaluation System - Based on Hangzhou High Star Hotel Reviews Data from Tripadvisor.com
}

\author{
Miao Zhangwei \\ School Of Tourism And Urban-Rural Planning, Hangzhou, Zhejiang, China \\ 1970172953@qq.com
}

Keywords: Online Reviews; Evaluation System; Customer Satisfaction

\begin{abstract}
In recent years, with the rapid development and popularization of the mobile Internet, online booking and purchasing of products has become a consumption habit. In order to better meet the needs of consumers, many online booking and purchasing platforms provide consumers with a free and anonymous evaluation model of products. Based on this, a huge amount of product evaluation information has been formed on major booking platforms. This paper uses the review information of Hangzhou high-star hotel in the third-party platform of Tripadvisor.com as the data source of Hangzhou customer satisfaction evaluation system, and using the method text analysis on big data to sort and analyze the collected hotel review information. Then, the Bayesian network model is used to construct the structural learning and parameter learning for each index to determine the factors affecting customer satisfaction. The research shows that the customer satisfaction evaluation system based on the online comment text is more accurate and scientific than the traditional one, which is beneficial to the hotel's accurate evaluation of customer satisfaction and customer demand identification.
\end{abstract}

\section{酒店顾客满意度评价体系研究}

一一基于 Tripadvisor.com 的杭州高星级酒店评论数据

\author{
缪章伟 \\ 浙江工商大学旅游管理系，杭州，浙江，中国 \\ 1970172953@qq.com
}

关键词: 在线评论; 评价体系; 顾客满意度

摘要：随着移动互联网的飞速发展和普及，线上预定购买产品已经成为一种消费习惯。许多 在线预订购买平台为了更好的满足消费者需求，为消费者提供了对产品进行自由、匿名的评 价模式。本文利用 Tripadvisor.com 第三方平台中杭州高星级酒店的点评信息, 作为杭州的顾 客满意度测评体系的数据基础, 之后运用贝叶斯网络对各因素进行结构学习和参数学习的构 建, 通过贝叶斯网络构建各维度和对应因素之间的相互关系, 确定顾客满意度影响因素。研 究表明, 基于在线评论文本构建的顾客满意度评价体系较传统的方式更为精准、科学, 有利 于酒店对顾客满意度的准确评价，具有一定的实践意义。

\section{1. 引言}

移动互联网的快速发展不仅提高人们生活的便捷程度, 也改变了人们消费的方式。在旅 行过程中，人们会选择 OTA 等线上平台对旅行产品和服务进行预订和了解。在整个网上旅游 
预订中, 包括网上预订机票、酒店、火车票和旅游度假产品等, 酒店预订是整个旅行预订中 的重要内容, 占旅游预订中的 $30.3 \%$ 。在线预订购买平台不仅为顾客提供预订和购买产品服 务, 也为消费者提供了对产品进行自由、匿名的评价模式。这种评价的模式, 受到消费者的 青睐, 吸引着众多消费者在购买产品服务后, 留下对产品的大量评论和信息。消费者在线预 订购买产品后所留下的线上评论不仅改变了顾客对产品信息查询和分享的方式，也改变了人 们获取产品信息的渠道从传统的广告等方式转变为顾客在平台中自由交流、评论的方式。

目前，移动互联网已成为消费者预定产品服务和获取产品消息的重要途径。越来越多的 顾客渐渐习惯预订酒店前, 关注酒店官网或第三方平台上其他消费者的评价, 并在入住后对 酒店进行评价。这些评论很大程度上反映了顾客对酒店的期望和体验感受, 从而体现出消费 者对酒店的满意程度 [1]。消费者在网络平台上的在线评论是公开的, 不论对酒店是正面评价 或是负面评价, 这都将正面影响其他潜在消费者对产品的印象, 也将直接影响到产品品牌的 口碑和盈利。

\section{2. 在线评论的相关研究}

\section{1 在线评论与顾客满意度的相关性}

目前，有关在线评论与酒店顾客满意度之间的关系存在争议。在线评论是顾客消费后的 体验与感受, 但其是否真的能够代表顾客对酒店的满意水平, 需要进行考证。有研究者认为 在线评论很大程度上反映了消费者消费的体验感知和期望的比较, 充分体现了消费者对酒店 服务质量的关注重点和感知评价，在酒店竞争中能起很大效果，但不能完全客观的说明消费 者是否满意。因为顾客不同的评分标准会很大程度上影响评分的高低[2], 并且每位顾客对于 酒店的重视方面与关注点都不尽相同, 也没无法完整的对酒店多方面进行评价。所以在线评 论是无法直接说明酒店顾客对于酒店满意度程度。

在线评论可作为一种文本数据库, 为研究者提供大量的文本信息, 通过对文本数据的整 理笁选和分析, 并根据不同的研究方法, 制定不同类型酒店的满意度测量体系, 以此测定顾 客对酒店的多方面的满意情况。吴必虎、熊伟等通过对国内外的酒店网络评论分析, 发现对 于各项基础设施, 国外的评价关注度远高于国内评价, 但满意度却远低于与国内评价, 国外 评价的各项满意度都低于国内水平[3]。Zheng Xiang、Zvi Schwartz 等采用了一种文本分析方 法, 从 Expedia.com 提取大量消费者评论, 以分析解构酒店客户体验, 并考察其与满意度评 分之间的关联[4]。丁于思、肖轶楠通过网站上的点评数据, 发现挂牌五星级酒店总体顾客满 意度为中等偏上水平, 最高分与最低分差距比较大, 并且从酒店五个单维度满意度进行分析 [1]。

从当前在线评论与酒店顾客满意度之间关系的研究看, 不难发现更多的研究者是将在线 评论作为一种数据进行处理, 并无法直接客观的表明顾客对酒店的满意水平, 需要通过不同 的研究方法和研究思路, 探索在线评论对顾客满意度的积极影响。

\section{2 在线评论对酒店业产生重要价值}

当前, 企业与研究人员对在线评论非常重视, 大量的在线评论逐渐体现出它的真正价值。 从实际运营的角度来看，对于酒店，消费者的在线评论已经成为酒店获取消费者真实感受的 一种重要渠道, 酒店可以根据在线评论中顾客提出的建议改善自己的经营方式, 或者改善产 品与服务的不足之处。在学术的层面, 不论是国内的学者还是国外的学者, 都开始将消费者 的在线评论作为一种数据的来源, 进行收集、分类和分析, 在此基础上对相关问题进行更深 层次的探讨。酒店的在线评论既是一种工具, 也是一种资源。在线评论是基于消费者在网络 空间的平等性、虚拟性等特点下，在消费结束后将自己真实的消费体验、感受以及心得用简

(1)第 43 次《中国互联网络发展状况统计报告》 
短的评价等形式的表现。这些酒店消费评论是消费者本身不用受到其他外在因素的干扰，用 亲身的体会对在酒店所消费的内容进行相应的评价, 从而这些在线评论具有真实性与客观性, 也提高了研究的准确性和可信度。

在相关平台阅读查看酒店的评价已成为顾客在预定酒店前的一种习惯，其主要目的是为 了方便、质量、社会保障和降低风险[5]。这些对酒店的评价不仅会影响其他消费者对酒店的 感知, 还会影响顾客的消费行为以及未来酒店的客房销售状况。大多数顾客阅读的评论时一 般不会超过前两页 [6], 并且近期的评论对顾客的影响也要大于之前的评论[7]。对于酒店而言, 一次正面的在线评论能为酒店带来一些益处，而一次负面的在线评论一般不会对酒店造成损 失[8]，但对于顾客，负面的评论可能会使顾客对酒店的认知产生了不利的影响 [9]。

在线评论可以为研究酒店提供很多有用的素材和数据, 它所拥有的价值并没有被充分的 挖掘和利用。在线评论不止能成为被研究的对象, 还能够为酒店或者学者提供有效的数据, 提供消费者的对酒店各方面感官和态度的评价。消费者消费后体验得出了这些评论，其他潜 在消费者可以基于这些评论就做出最适合自己的选择, 相应的企业也应该充分挖掘和利用这 些信息进行更好决策[10]。许多企业已经意识到评论文本数据的潜在价值，通过这些数据能 够更加深入了解顾客以及分析指导管理的决策[11]。目前酒店在线评论的广泛使用，对于酒 店行业而言, 是一个发展的机会, 而不是一种威胁[12]。

\section{3. 基于在线评论的酒店顾客满意度评价体系构建}

查找酒店评语，从中提炼顾客对酒店提的较多或关注方面的高频词，根据分类总结得出 的高频词分类因素和维度设计顾客满意度影响因素初始问卷, 并发放。之后采用贝叶斯网络, 分析各因素可能对顾客满意度的影响概率, 并将影响概率较低或不确定较高的影响因素排除, 确定最终的顾客满意度影响因素，构建出测评体系。

\section{1 在线评论分析}

目前, 有关酒店预定评论的网站或平台数量繁多, 为了获取准确和质量高的评价样本, 综合考虑后, 本文最终确定以 tripadvisor.com 第三方网站平台为评论数据的主要来源。

通过对 tripadvisor.com 网站中的杭州高星级酒店的 33945 条点评数据抓取和整理, 并对 整理过后的点评数据完成分词和同义词合并的处理。运用 ROST CM6 软件对经过处理的文本 数据进行分析, 从中得到可能成为杭州高星级酒店顾客满意度影响因素的因子。选用 ROST CM6 软件中的高频词提取功能, 对分词过后的评论汇总文件进行提取高频词, 提取前 300 的 高频词词汇, 再经过笛选和过滤, 剩余有效高频词 117 个。之后根据词意和词性对有效高频 词汇进行分类，提炼总结出二十八个高频词分类因素和六个高频词划分维度，如表 1 。

表 1 基于在线评论的高频词分类

\begin{tabular}{l|c|c}
\hline \multicolumn{1}{c|}{ 高频词汇 } & 高频词分类 & 概括归类 \\
\hline 位置、西湖、市中心、机场、距离、市区、位于、火车站 & 地理位置 & 区位环境 \\
附近、靠近、周围、购物、周边、地段、景点、景区、湖边、商场、 & & \\
风景、边上、广场、景色、旁边 & 边环境 & \\
交通、打车、步行、出门、方便、公交车、公交、出行 & 交通 & \\
\hline 早餐、免费、自助 & 早餐质量 & 餐饮 \\
餐厅 & 餐厅环境 & \\
口味、好吃、味道 & 食品味道 & \\
品种、种类、丰富 & 食品种类 & \\
自助餐 & 自助餐质量 & \\
酒廊 & 酒廊 & \\
\hline 接待、行李 & 礼宾服务 & 服务质量 \\
前台 & 前台服务 & \\
\hline
\end{tabular}




\begin{tabular}{l|c|c}
\hline 打扫、干净、卫生、整洁 & 清扫服务 & \\
礼貌、温馨、态度、热情、服务态度、贴心、亲切、主动 & 服务态度 & \\
速度、及时、效率 & 服务效率 & \\
细节、周到、到位 & 服务细节 & \\
\hline 电视、空调、电脑、上网、陈旧、房间设施 & 客房设施 & 客房环境 \\
设计、阳台、宽敞、窗户、行政 & 客房设计 & \\
卫生间、浴缸、浴室 & 客房浴室 & \\
隔音、声音、效果、安静 & 隔音效果 & \\
一次性、用品 & 生活用品 & \\
\hline 电梯、设施、硬件、齐全、楼层 & 酒店设施 & 酒店环境 \\
风格、装修、商务、特色、豪华、装潢、档次、繁华、度假、星级 & 装饰及风格 & \\
大厅、大堂、环境 & 大堂环境 & \\
空气、气味 & 空气质量 & \\
游泳池 & 康乐设施 & \\
停车、停车场 & 停车场 & \\
舒适、舒服、整个、整体、总体 & 整体舒适 & \\
\hline 价格、性价比、价位、便宜、值得 & 性价比 & 价格 \\
\hline
\end{tabular}

\section{2 确定顾客满意度影响因素}

通过对专家进行访问，依据二十八个高频词分类和六个高频词划分维度设计初始满意度 影响因素问卷, 针对在杭州高星级酒店消费过的顾客, 开始“杭州高星级酒店顾客满意度影响 因素问卷调查”。研究共发 180 份, 有效回收问卷 157 份, 有效率为 $87.2 \%$ 。从问卷收集的完 整数据样本中, 分别将六个一级因素维度和二十八个对应二级因素带入贝叶斯网络分析中, 建立贝叶斯网络的过程将选用 Netica 软件。

在 Netica 软件中建立一个新的贝叶斯网络窗口，调用一级因素维度变量以及对应二级因 素的相关数据集, 采用软件中网络结构学习模块和参数学习模块的功能, 创建贝叶斯网络。 每个因素中 1、2、3 为因素对顾客满意度存在影响的状态 (1 不存在影响; 2 不确定; 3 存在 影响)。

采用问卷的方式收集数据, 通过顾客对各变量能否影响对酒店满意度感知的数据构建贝 叶斯网络结构和参数, 得出有向无环图和各节点的条件概率表。之后采用贝叶斯网络预测分 析的概率推理方法, 证明一级影响因素与对应二级因素之间存在的关系以及变化, 例如节点 $\mathrm{A}$ 为节点 $\mathrm{B}$ 的父节点, 当 $\mathrm{A}$ 处于某一状态时, $\mathrm{B}$ 节点所产生的概率值变化 [13]。在文中, 两 个具有相关关系的满意度影响因素, 得出结构和参数后, 通过推理功能可以十分明确表明两 节点间的关系程度, 然后根据概率值了解节点对顾客满意度的影响变化, 将影响度较低和不 确定较高的顾客满意度影响因素进行排除, 得出最后的顾客满意度测评体系。

本文将通过专业的贝叶斯网络构建软件 Netica，构建出满意度指标的网络结构以及网络 参数。Netica 软件是一款功能强大, 简单, 易于操作和使用的贝叶斯网络工具。此软件可以 根据需求自行构建贝叶斯网络或运用软件中的程序运算方法, 将处理完善的数据直接学习和 构建贝叶斯网络。

在 Netica 软件中建立一个新的贝叶斯网络窗口，根据因素标注不同的序号 U1-U34，调用 一级因素维度变量的相关数据集, 创建贝叶斯网络。以 U1 因素维度区位环境, 及对应因素 U7 地理位置、U8 周边环境和 U9 交通为例。通过 Netica 构建贝叶斯网络, 再运用预测分析 的方法, 得出一级因素维度“区位环境”对应的二级因素地理位置、周边环境和交通的结构学 习和参数学习。如图 1 和表 2 中得出 $\mathrm{P}(\mathrm{U} 7=3 \mid \mathrm{U} 1=3)=88.356 \%, \mathrm{P}(\mathrm{U} 8=3 \mid \mathrm{U} 1=3)=86.301 \%$, $\mathrm{P}(\mathrm{U} 9=3 \mid \mathrm{U} 1=3)=90.411 \%$, 其表示为当因素维度区位环境对顾客满意度存在影响时, 那么因 素地理位置对顾客满意度存在影响的概率也由 $82 \%$ 改变为 $88.356 \%$; 周边环境对顾客满意度 的影响由 $81.8 \%$ 改变为 $86.301 \%$ 以及交通对顾客满意度存在影响的概率由 $84.4 \%$ 改变为 $90.411 \%$ 。从数据中可以看出当一级因素维度对顾客满意度的影响发生变化时, 对应的三个二 
级因素也会发生变化, 且与对应因素维度的变化产生正相关变化, 由此可得一级因素维度区 位环境与二级因素地理位置、周边环境和交通之间存在相互依赖关系。但由于 $P(U 7=3 \mid U 1=2)$ $=25 \%, \mathrm{P}(\mathrm{U} 7=, 1 \mid \mathrm{U} 1=2)=37.5 \%, \mathrm{P}(\mathrm{U} 7=, 2 \mid \mathrm{U} 1=2)=37.5 \%$, 表现为当指标维度 U1 处于不确 定对顾客满意度产生影响时, 且 $P(U 7=, 1 \mid U 1=2)>P(U 7=3 \mid U 1=2)$ 因素 $U 7$ 对顾客满意度产 生影响的概率要低于不产生影响, 所以 U7 地理位置因素对顾客满意度存在影响的概率较低, 不确定性较高, 故此将此因素排除顾客满意度体系外。

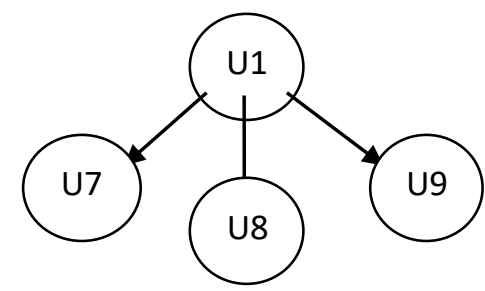

图 1 区位环境中构成的网络结构

表 2 区位环境中各因素的条件概率表

\begin{tabular}{|l|ccc|}
\hline $\mathrm{U} 1$ & $\mathrm{P}(\mathrm{U} 1=1)$ & $\mathrm{P}(\mathrm{U} 1=2)$ & $\mathrm{P}(\mathrm{U} 1=3)$ \\
\hline & 0.0125 & 0.0875 & 0.9 \\
\hline
\end{tabular}

\begin{tabular}{|l|lll|}
\hline U1/U7 & 1 & 2 & 3 \\
\hline 1 & 0.5 & 0.25 & 0.25 \\
2 & 0.375 & 0.375 & 0.25 \\
3 & 0.00685 & 0.10959 & 0.88356 \\
\hline
\end{tabular}

\begin{tabular}{|l|lll|}
\hline U1/U8 & 1 & 2 & 3 \\
\hline 1 & 0.50 & 0.25 & 0.25 \\
2 & 0.25 & 0.3125 & 0.4375 \\
3 & 0.02055 & 0.11644 & 0.86301 \\
\hline
\end{tabular}

\begin{tabular}{|l|lll|}
\hline U1/U9 & 1 & 2 & 3 \\
\hline 1 & 0.25 & 0.50 & 0.25 \\
2 & 0.3125 & 0.375 & 0.3125 \\
3 & 0.0137 & 0.08219 & 0.90411 \\
\hline
\end{tabular}

以同样的贝叶斯网络分析方法，分别对六个因素维度和对应因素进行网络构建。由贝叶 斯网络预测分析中得出在指标维度处于不同状态下, 指标在某一条件下的概率值, 因此也得 出因素对顾客满意度存在影响的概率值，其中由于地理位置 U7、酒廊 U15、客房设计 U23 和大堂环境 U29 二级因素, 在对应因素维度处于不确定对顾客满意度存在影响状态时, 四个 二级因素对顾客满意度不存在影响的概率大于存在影响的概率, 由此说明四个二级因素可能 对顾客满意度不存在影响的概率更高, 故此将四个二级因素移除。

\section{3 确定顾客满意度评价体系}

通过以上对每个一级因素维度进行的贝叶斯网络分析, 发现 U7、U15、U23、U29 四个 因素是否会能够成为顾客满意度评价体系的指标, 还需要进一步的研究, 最终确定六个一级 指标及对应二十四个二级顾客满意度影响因素, 分析结果的顾客满意度评价体系如表 3:

表 3 杭州高星级酒店顾客满意度评价指标体系

\begin{tabular}{|c|c|c|c|c|c|c|c|}
\hline & 一级指标 & & 二级指标 & & 一级指标 & & 二级指标 \\
\hline$\overline{\mathrm{U} 1}$ & 区位环境 & $\begin{array}{l}\text { U8 } \\
\text { U9 }\end{array}$ & $\begin{array}{l}\text { 周边环境 } \\
\text { 交通 }\end{array}$ & $\mathrm{U} 4$ & 客房环境 & $\begin{array}{l}\mathrm{U} 22 \\
\mathrm{U} 24 \\
\mathrm{U} 25 \\
\mathrm{U} 26\end{array}$ & $\begin{array}{l}\text { 客房设施 } \\
\text { 客房浴室 } \\
\text { 隔音效果 } \\
\text { 生活用品 }\end{array}$ \\
\hline $\mathrm{U} 2$ & 餐饮 & $\begin{array}{l}\text { U10 } \\
\text { U11 } \\
\text { U12 } \\
\text { U13 } \\
\text { U14 }\end{array}$ & $\begin{array}{l}\text { 早餐质量 } \\
\text { 餐厅环境 } \\
\text { 食品味道 } \\
\text { 食品种类 } \\
\text { 自助餐质量 }\end{array}$ & & 酒店环境 & $\begin{array}{l}\text { U27 } \\
\text { U28 } \\
\text { U30 } \\
\text { U31 } \\
\text { U32 } \\
\text { U33 }\end{array}$ & $\begin{array}{l}\text { 酒店设施 } \\
\text { 装饰及风格 } \\
\text { 空气质量 } \\
\text { 康乐设施 } \\
\text { 停车场 } \\
\text { 整体舒适度 }\end{array}$ \\
\hline & 服务质量 & $\begin{array}{l}\text { U16 } \\
\text { U17 }\end{array}$ & $\begin{array}{l}\text { 礼宾服务 } \\
\text { 前台服务 }\end{array}$ & & 价格 & U34 & 性价比 \\
\hline
\end{tabular}




\section{4. 评价体系的实际案例运用}

顾客满意度评价体系设计是否合理, 将直接影响测评结果的真实性和有用性。因此, 文 中将采用实际案例的运用, 结合实际情况, 验证顾客满意度体系的合理性。本文所选择的案 例酒店为杭州的 JW 万豪酒店, 原因有三, 一是 JW 万豪酒店作为国内外知名的高端酒店, 对高星级酒店消费群体中具有一定的影响力和知名度; 二是万豪国际酒店管理集团拥有丰富 的管理经验，更能达到顾客对于高星级酒店产品与服务的期望；三是 JW 万豪酒店在杭州已 运营九年, 在品牌和口碑具有一定积累, 获取顾客的在线评论的准确性和真实性相较于其他 高星级酒店更高。所以文中选择杭州 JW 万豪酒店为测评体系的检验与实行案例酒店。

\section{1 酒店在线评论数据收集}

根据的顾客满意度指标, 从 Tripadvisor.com 中找出 JW 万豪酒店中的 988 条评论中采用 语义分析得出相关数据, 如表 4:

表 4 在线评论语义分析样例

\begin{tabular}{|c|c|c|c|c|}
\hline 顾客评论 & 评论内容 & 一级指标 & 二级指标 & 满意程度 \\
\hline 位置真棒, 西湖边上, & 位置真棒, 西湖边上。 & 区位环境 & 周边环境 & 满意 \\
\hline $\begin{array}{l}\text { 住 } 26 \text { 层。房间装修风 } \\
\text { 枚喜欢很性化 }\end{array}$ & 卫牛间设施跟住过的宇波 & & & \\
\hline 生间设施跟住过的宁 & 万豪有太大差距。 & 客房环境 & 客房浴室 & 非常不满意 \\
\hline $\begin{array}{l}\text { 波万豪有太大差距。酒 } \\
\text { 店的餐厅价格太贵, 人 }\end{array}$ & 打车不便。 & 区位环境 & 交通 & 不满意 \\
\hline $\begin{array}{l}\text { 均居然差不多要 } 300 \\
\text { 左右。打车不便。太不 } \\
\text { 划算, 性价比真心低。 }\end{array}$ & 性价比真心低。 & & 性价比 & 非常不满意 \\
\hline & & & & \\
\hline
\end{tabular}

通过对满意度影响因素和满意度的状态, 收集的 JW 万豪酒店在线评论中所出现的频次 数据如表 5 。

表 $5 \mathrm{JW}$ 万豪酒店评价集

\begin{tabular}{|c|c|ccccc|}
\hline 一级指标 & 二级指标 & 非常不满意 & 不满意 & 一般 & 满意 & 非常满意 \\
\hline 区位环境 & 周边环境 & 0 & 2 & 3 & 63 & 44 \\
& 交通 & 0 & 9 & 1 & 48 & 27 \\
\hline 餐饮 & 早餐质量 & 2 & 5 & 6 & 75 & 86 \\
& 餐厅环境 & 1 & 1 & 0 & 21 & 44 \\
& 食品味道 & 0 & 1 & 7 & 63 & 76 \\
& 食品种类 & 0 & 11 & 9 & 60 & 51 \\
& 自助餐质量 & 0 & 2 & 1 & 22 & 16 \\
\hline 服务质量 & 礼宾服务 & 4 & 5 & 4 & 25 & 48 \\
& 前台服务 & 4 & 10 & 6 & 42 & 66 \\
& 清扫服务 & 5 & 5 & 7 & 59 & 111 \\
& 服务态度 & 3 & 3 & 4 & 105 & 233 \\
& 服务效率 & 5 & 9 & 4 & 44 & 114 \\
& 服务细节 & 4 & 6 & 4 & 65 & 196 \\
\hline 客房环境 & 客房设施 & 5 & 14 & 5 & 69 & 53 \\
\hline
\end{tabular}




\begin{tabular}{|c|c|ccccc|}
\hline \multirow{7}{*}{} & 客房浴室 & 2 & 7 & 4 & 40 & 47 \\
& 隔音效果 & 6 & 5 & 2 & 22 & 24 \\
& 生活用品 & 2 & 3 & 3 & 23 & 22 \\
\hline 酒店环境 & 酒店设施 & 2 & 7 & 5 & 33 & 30 \\
& 装饰及风格 & 0 & 6 & 5 & 37 & 21 \\
& 空气质量 & 1 & 0 & 2 & 11 & 12 \\
& 康乐设施 & 4 & 8 & 5 & 30 & 31 \\
& 停车场 & 0 & 0 & 2 & 19 & 11 \\
& 整体舒适 & 1 & 1 & 9 & 33 & 117 \\
\hline 价格 & 性价比 & 1 & 9 & 17 & 18 & 45 \\
\hline
\end{tabular}

\section{2 顾客满意度指数计算结果}

顾客满意度测评体系其最终目的是为了了解顾客对于酒店的各方面的满意程度，因此需 要通过文本分析对在线评论进行量化。根据上述对满意度状态的五种状态分类, 对参评酒店 中涉及的二级指标进行不同满意程度的数量统计, 并依据满意度的五种状态分别得到五种得 分分数, 即 $\{$ 非常不满意, 不满意, 一般, 满意, 非常满意 $\}$ 满意状态对应分数向量 $\{20,40,60,80,100\}[1]$, 最终采用加权平均的计算方法得出结果。

$$
S_{i}=20 \cdot U_{i} \text { 非常不满意 }+40 \cdot U_{i} \text { 不满意 }+60 \cdot U_{i} \text {-般 }+80 \cdot U_{i} \text { 满意 }+100 \cdot U_{i} \text { 非常满意 }
$$

根据公式, 通过加权平均的算法, $\mathrm{U}_{\mathrm{i}}$ 为指标满意度状态的百分比, $\mathrm{S}$ 为顾客满意度指数。

JW 万豪酒店通过计算得出酒店整体顾客满意度指数为 86.98 , 其中“区位环境”的顾客满 意度指数为 84.58 , “餐饮”顾客满意度指数为 87.28 , “服务质量”的顾客满意度指数为 89.84 , “客房环境”顾客满意度指数为 81.62 , “酒店环境”顾客满意度指数为 85.68 和“价格”顾客满意 度指数为 81.56 。

根据计算得出的顾客满意度指数，顾客对于酒店处于满意与非常满意之间呈现了较好的 顾客满意度情况, 说明顾客对于酒店的各方面有较高的认可, 这与 JW 万豪酒店在 Tripadvisor.com 网站中杭州市酒店排名第 15 名相契合, 拥有较高的顾客满意状况。

\section{5. 结束语}

在我国的酒店行业, 目前高星级酒店的数量增加迅速, 且部分地区产品结构不均衡, 导 致市场竞争激烈。同时, 由于住宿业态的多样化趋势显著, 各类住宿产品的出现对国内传统 高星级酒店的冲击也比较大，使得高星级酒店持续面临激烈的市场竞争环境。高星级酒店想 要在激烈的市场竞争中长远发展, 需要更精准的面向顾客需求, 提高顾客满意度, 所以更科 学有效的顾客满意度评价体系是高星级酒店面临的重要问题。本文在前人的研究基础上, 利 用在线评论大数据中找出顾客对酒店关注较多的层面, 从而了解顾客对于酒店的关注重点和 需求, 以此为基础构建出顾客满意度评价体系。该体系从顾客的角度出发, 拥有较高的合理 性和科学性，对酒店业具有一定的实践意义和参考价值。

\section{References}

[1] Si, DING Yu and Nan, XIAO Yi, Customer Satisfaction Evaluation of Five-Star Hotels Based on Online Comments, Economic Geography, vol.34, pp. 182-186+192, 2014.

[2] GAO Bao Jun, SUN Han Lin and WANG Han Ning, Influence of Online Reviews on Hotels'Full-occupancy Rates, Tourism Tribune, vol.31, pp. 109-117, 2016.

[3] XIONG Wei, GAO Yang and WU Bi-hu,A Comparison Research of Online Commentary about Service Quality in Sino- foreign International High- rated Chain Hotels, Economic Geography, vol.32, pp. 160-165, 2012. 
[4] Xiang, Z., et al., What can big data and text analytics tell us about hotel guest experience and satisfaction? International Journal of Hospitality Management, vol.44, pp. 120-130, 2015.

[5] Kim, E.E.K., A.S. Mattila and S. Baloglu, Effects of Gender and Expertise on Consumers' Motivation to Read Online Hotel Reviews. Cornell Hospitality Quarterly, vol.52, pp. 399-406, 2011.

[6] Pavlou, P.A. and A. Dimoka, The Nature and Role of Feedback Text Comments in Online Marketplaces: Implications for Trust Building, Price Premiums, and Seller Differentiation. Information Systems Research, vol.17, pp. 392-414, 2006.

[7] Dellarocas, C., The Digitization of Word of Mouth: Promise and Challenges of Online Feedback Mechanisms. Management science: Journal of the Institute of Management Sciences, vol.49, pp. 1407-1424, 2003.

[8] Vermeulen, I.E. and D. Seegers, Tried and tested: The impact of online hotel reviews on consumer consideration. Tourism Management, vol.30, pp. 123-127, 2009.

[9] Browning, V., K.K.F. So and B. Sparks, The Influence of Online Reviews on Consumers' Attributions of Service Quality and Control for Service Standards in Hotels. Journal of Travel \& Tourism Marketing, vol.30, pp. 23-40, 2013.

[10]XU Feng, Study on the Effect of Online Information on the Online Hotel Bookingsin Different Types of Chinese Cities_—Based on the Ctrip's Data, Journal Of Business Economics, vol. 261, pp. 73-80, 2013.

[11]Phillips-Wren, G. and A. Hoskisson, An analytical journey towards big data. Journal of Decision Systems, vol. 24, pp. 87-102, 2015.

[12]Litvin, S.W., R.E. Goldsmith and B. Pan, Electronic word-of-mouth in hospitality and tourism management. Tourism Management, vol. 29, pp. 458-468, 2008.

[13]ChenJin, Jiang Zhengkai and Fu Jingqi, Construction of self-learning Bayesian network based on Netica. Journal Of Electronic Measurement And Instrumentation, vol. 30, pp. 1687-1693, 2016. 